\title{
Agent-based analysis of dynamic access ranges to the distribution network
}

\author{
S. Mathieu, D. Ernst, B. Cornélusse \\ University of Liège, Belgium
}

\begin{abstract}
There is a need to clearly state an interaction model that formalizes interactions between actors of the distribution system exchanging flexibility. In previous works we quantitatively evaluated the performance of five interaction models devised with industrial partners using the agent-based testbed DSIMA. Simulation results showed that these interaction models relying on active network management suffer from a lack of coordination between the distribution and the transmission system operator, activating flexibility simultaneously in opposite directions. This paper introduces a new interaction model fixing this issue based on dynamic access bounds to the network changing throughout the day and preventing the activation of flexibility leading to congestions. This new interaction model is implemented in DSIMA and compared to a model restricting the grid users to a very restrictive but safe access range. Results show that this new model allows to safely increase by $55 \%$ the amount of distributed generation in the network.
\end{abstract}

Index Terms-distribution networks, market design, load flexibility, demand-side management, agent-based model, smart grid

\section{INTRODUCTION}

A current trend in distribution systems is to use the flexibility of generation means (e.g. curtailment) and consumers (e.g. load shifting), in addition to regular control assets such as tap changers, for balancing or local congestion management. For instance, distribution system operators (DSOs) rely on active network management to anticipate and correct, using flexibility, operational limits violations when this is more economical or easier to perform than straightforward grid reinforcement. However, there are many possibilities to organize interactions between the actors of the system when flexibility comes into play. Thus, there is a need first to clearly state the interaction models that formalize these interactions, and second to set up a way for comparing their economical and technical merit.

In previous works we evaluated the performance of five interaction models devised with industrial partners using the open-source agent-based testbed DSIMA [1]. The scope of the interaction models covers several stages from day-ahead exchanges of flexibility until settlement. In DSIMA, the actors of the system are modeled as individual agents that solve a nested sequence of optimization problems to take their decision in order to maximize their individual objective. At each decision stage the problem is constrained by the decisions taken at the previous stages, and subsequent recourse possibilities are taken into account. The agents considered are the DSO, the transmission system operator (TSO), producers and retailers. The impact of the agent's decisions is evaluated through a measure of the social welfare, the share of the welfare between the actors, and a measure of the service level.

Simulation results on an expected system in 2025 show that the interaction models proposed in [1] relying on active network management, in the way they have been stated, suffer from a lack of coordination between the DSO and the TSO. The current paper introduces a new interaction model fixing this issue. The model is based on dynamic access bounds to the network changing through the day and computed by the DSO. These bounds are computed using baseline proposals from the grid users at the medium voltage level. This new interaction model is implemented in DSIMA [1] and compared to the previous safest proposal, an interaction model restricting the grid users to a very restrictive but safe access range.

The paper is organized as follows. Section II reviews the relevant literature. A summary of the agent-based testbed DSIMA is given in Section III. The coordination issue arising in the interaction models analyzed in [1] is explained in more details in Section IV, which also describes a new interaction model that solves this issue. Section V describes the implementation of these interaction models in DSIMA. Test results are reported in Section VI, and Section VII concludes.

\section{LITERATURE REVIEW}

The common method to guarantee that distribution networks can be safely operated is to restrict the access of distributed generation to the distribution network. The power systems literature already contains many solutions to operate the network considering the curtailment of distributed generation units [2]-[5]. Regulated non-firm generation access contracts are introduced in [3] where units are subject to curtailing and/or shedding, depending on the requirements from the network operation. The DSO may define the operating margins of the units with the latter contract to reach a safe operating point. A method to determine these margins is proposed and demonstrated in [3]. Article [2] reviews renewable energy curtailment schemes and principles of access contracts. Three potential strategies for congestion management based on the generic interaction model of [4] are presented in [5]: distribution grid capacity market, advance capacity allocation, dynamic grid tariff. The authors provide a qualitative analysis of their models. Their model advance capacity allocation is close to the interaction model 6 proposed in this paper.

On the demand side, flexibility is mostly obtained through dynamic pricing [6] or direct control of the loads [7], [8]. Both methods have drawbacks. Dynamic pricing exposes the end-user of the load to volatile prices, which may not be 
appropriate for retail consumers [9]. Dynamic pricing also leaves uncertainty on the quantity of flexibility that is actually deployed and therefore on the cost of this flexibility. This uncertainty does not arise with the direct control of the load but requires direct access to the controlled loads which implies challenging control issues and intrusive equipment. One alternative to these two methods is to rely on dynamic fuses at the network connections and leave to the connected equipment the optimization of its consumption in order to satisfy the power limit of the fuse [9]. An aggregator may control the fuse limit in case the total net load should be limited to ensure the well functioning of the network [10]. This kind of load control perfectly fits with the interaction model presented in Section IV.

\section{DEFINITION OF INTERACTION MODELS AND THEIR IMPLEMENTATION IN DSIMA}

This section recalls the main elements of the system modeled in DSIMA. More details can be found in the full publication [1]. The analysis is centered on the short-term operation of a medium-voltage network. In the scope of this paper, we consider the short-term flexibility exchanges in an operational planning phase. The actors simulated in DSIMA are the DSO, the TSO, producers and retailers, and may fulfill more than one role, depending on the interaction model. The roles considered are balance responsible parties (BRP), flexibility services providers (FSP) and flexibility services users (FSU). To operate its network, the DSO may act as a FSU. The TSO is, from the point of view of this simulation, a FSU with given needs of flexibility services. Producers and retailers both act simultaneously as BRPs, FSPs and FSUs. The interaction models implemented in DSIMA follow the procedure below.

1) DSO analysis and TSO imbalance settlement are based on reference baselines for every bus. Right after the clearing of day-ahead energy market, BRPs submit their reference baselines to the DSO and the TSO.

2) The DSO assesses the state of the system and announces its flexibility needs to the FSPs. Other FSUs also announce their needs to the FSPs.

3) FSPs provide flexibility offers sequentially to each potential FSU, the first FSU being the DSO.

4) FSUs contracts some flexibility offers for their needs.

5) Closer to real-time, FSUs request the activation of their flexibility services.

6) Right before real-time, each FSP optimizes its realization taking into account the request of its FSUs, the penalty incurred if a flexibility service is not provided and its imbalance with respect to its BRPs.

7) The distribution network is operated using these realizations taking last resort actions if necessary, such as shedding buses. If such actions are needed and FSPs did not provide their service to the DSO, the FSPs are penalized at a regulated tariff.

These interactions between the actors of the system are summarized in Fig. 1. The courses of the events is described from left to right going from short-term interactions to settlement. Each type of actor is represented by a horizontal line. Each vertical arrow represents an interaction between two types of actor. The actor on the head of the arrow receives the information from the ones at the tails of the arrow indicated by the circles. The flexibility platform is an intermediary between FSUs and FSPs to exchange flexibility information.

A grid user makes first a request to the DSO to get access to a range $\left[g_{a, n}, G_{a, n}\right]: g_{a, n} \leq 0 \leq G_{a, n}$. Based on these requested access bounds, the DSO computes safe access bounds $\left[b_{a, n}, B_{a, n}\right]: b_{a, n} \leq 0 \leq B_{a, n}$. The safe access range ensures that no congestion occurs if every grid user accesses to the network in the limits $\left[b_{a, n}, B_{a, n}\right]$. We assume that there is an access contract between a BRP $a$ and the DSO for each bus $n$ the BRP has access to. A contract specifies a full access range $\left[l_{a, n}, L_{a, n}\right]$ and a wider flexible access range $\left[k_{a, n}, K_{a, n}\right]$, cf. Fig. 2. Note that these bounds are constant with respect to time. The BRP can produce or consume without any restriction within the full access range $\left[l_{a, n}, L_{a, n}\right]$, but in the flexibility intervals $\left[k_{a, n}, l_{a, n}\right]$ and $\left[L_{a, n}, K_{a, n}\right]$ the DSO can order a restriction of the production or consumption if necessary. Following the practice of electrical European market design, it is the responsibility of the grid user to take into account its technical constraints to be able to satisfy the requests of the DSO. The link between $\left[b_{a, n}, B_{a, n}\right],\left[l_{a, n}, L_{a, n}\right]$ and $\left[k_{a, n}, K_{a, n}\right]$ is particular to each interaction model [1]. One simple method which is adopted for the simulation and was proposed in [1] is described in Section V. A more detailed definition of this procedure is outside the scope of this paper.

We strongly encourage the reader to get more information about the agent-based model DSIMA, designed to study quantitatively interaction models for the exchange of flexibility within the distribution network, in paper [1]

\section{NEW INTERACTION MODEL WITH DYNAMIC RANGES}

In the previously proposed interaction models, stakeholders are free to exchange flexibility services. However, if the profile of a BRP is within the flexibility intervals, it has to propose flexibility offers to the DSO via FSPs. The DSO acts as a standard FSU to contract and to activate the flexibility services. Since every FSU activates flexibility simultaneously, some actions may counterbalance the action of the DSO. Fig. 3 illustrates this issue on a small example where an action of the TSO counterbalances and action of the DSO which was supposed to solve a congestion. Assume that the flow exceeds the capacity of line 3 by $1 \mathrm{MW}$. To solve this issue, the DSO curtails a wind turbine by $1 \mathrm{MW}$. In the same time, assume that the TSO asks a storage unit to increase its production by 0.4 MW. The simultaneous activation of these flexibility services leads to a remaining congestion of $0.4 \mathrm{MW}$ even though every agent stays within its access bounds and satisfies its flexibility request. We can distinguish two situations:

- The storage unit is in its safe range: the DSO anticipates that the profile of the storage unit could deviate from its baseline, for instance to provide a service to the TSO; 


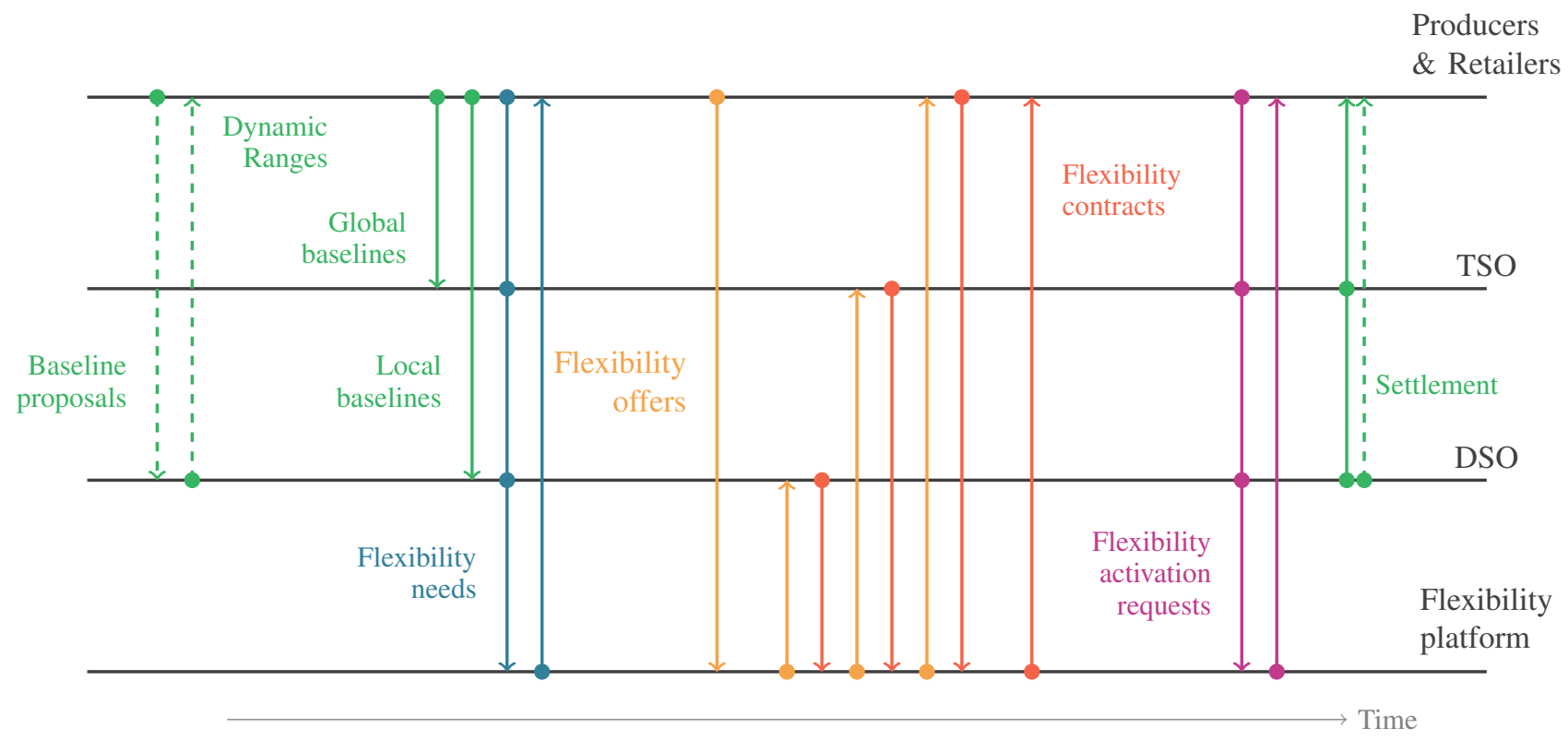

Fig. 1: Timeline of the interactions between the agents. The full lines correspond to interactions of the original DSIMA interaction models. The dotted lines correspond to new interactions of the proposed interaction model.

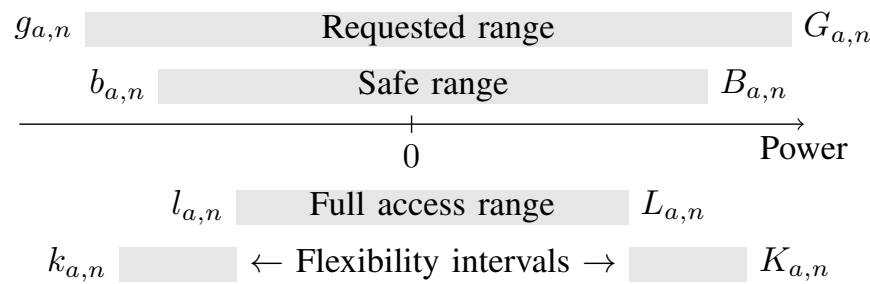

Fig. 2: Definition of access bounds.

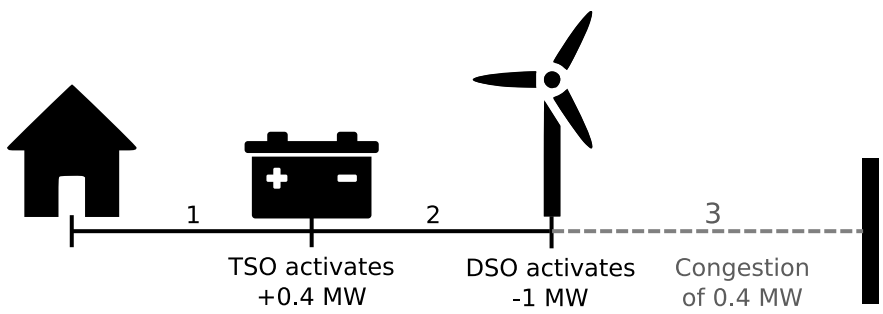

Fig. 3: Illustration of a flexibility activation coordination issue between the DSO and the TSO.

- The storage unit is in a flexibility interval: this flexibility interval is limited so that no flexibility activation can counterbalance the DSO actions.

The contribution of this paper lies in the proposal and the analysis of an interaction model with dynamic access ranges solving the previous issue. These ranges change each quarter based on the distribution network limitations computed by the DSO. These dynamic ranges are denoted $\left[d_{a, n, t}, D_{a, n, t}\right]$, and are constrained to be larger than the full access range. The procedure to obtain the dynamic ranges is the following.

1) BRPs provide baseline proposals that may belong to the full access range or the flexibility intervals.

2) Based on these proposals, the DSO computes the dynamic ranges so that its network is secure and communicates them to the BRPs.

3) BRPs submit new baselines, constrained to lie within the dynamic ranges, to the DSO and the TSO.

4) These baselines are used as reference for the provision of flexibility services. If the realization of a BRP violates the dynamic ranges, the BRP is penalized at a regulated tariff higher but of the same order of magnitude than the imbalance price.

These additional steps are represented by the dotted lines in Fig. 1. Coming back to our example, assume that the wind turbine has the safe range $[0,5]$ MW and provide the baseline proposal of $7 \mathrm{MW}$. The storage unit has a flexible range of $[-1,1]$ MW and a baseline proposal of $0 \mathrm{MW}$. To solve the congestion problem, the DSO grants the dynamic range $[0,6]$ MW to the wind turbine and $[-1,0]$ MW to the storage unit. As a result, the TSO cannot ask the storage unit to increase its production and the DSO prevents the congestion.

Note that a baseline proposal is mandatory to discourage a grid user to produce or consume outside of its dynamic range established on a definitive baseline. For instance, assume that a producer has a flexible range of $[5,8] \mathrm{MW}$ in one bus and decides to produce $8 \mathrm{MW}$, so that its baseline its $8 \mathrm{MW}$. After its computations, the DSO grants the dynamic range $[0,6] \mathrm{MW}$. The producer has to choose between paying an imbalance of 2 MW to the TSO or paying a penalty to the DSO. Now assume that the producer may sell a flexibility service to another BRP for a downward modulation of $1 \mathrm{MW}$, modulation for which the producer is remunerated. The producer has incentive to produce $7 \mathrm{MW}$, provide the flexibility service and pay only 
for $1 \mathrm{MW}$ of penalty to the DSO. Note that the producer is not responsible for any imbalance with this decision.

\section{DYNAMIC ACCESS RANGES COMPUTATION}

Prior to the day by day simulation of the system, the DSO computes the access bounds $\left[b_{a, n}, B_{a_{n}}\right]$ to its system for each agent $a$ and each bus $n$. We opt for the following procedure, which is performed only once for the whole simulation range, e.g. one year. The bounds for each bus and each agent $\left[b_{a, n}, B_{a, n}\right]$ are computed by solving the problem (1), which provides bounds that are as close a possible from the bounds that were requested by each agent, $\left[g_{a, n}, G_{a, n}\right]$. These bound are obtained by minimizing the sum of the auxiliary variables $\delta_{g}$ and $\delta_{G}$, representing the deviation from the requests, taking into account the operational limits of the system. Here, we consider the line capacities only in a network flow problem. The extension of the procedure to the classic optimal power flow problem considering reactive flow and voltage constraint would follow the same principle but would lead to non-linear optimization problems. Worst case conditions are considered through the auxiliary variables $\underline{f}_{n, m}$ and $\bar{f}_{n, m}$, but actual power flows are not. Note that in a real system, this procedure

\begin{tabular}{|c|c|}
\hline $\min \delta_{g}+\delta_{G}$ & (1a) \\
\hline \multicolumn{2}{|l|}{ subject to, $\forall n, n^{\prime} \in \mathcal{N}^{2}$, } \\
\hline$-C_{n, n^{\prime}} \leq \underline{f}_{n, n^{\prime}}, \bar{f}_{n, n^{\prime}} \leq C_{n, n^{\prime}}$ & $(1 b)$ \\
\hline \multicolumn{2}{|l|}{$\forall n \in \mathcal{N}$} \\
\hline$\sum b_{a, n}=\sum \underline{f}_{n, n^{\prime}}$ & $(1 \mathrm{c})$ \\
\hline$\sum_{a \in \mathcal{A}(n)} B_{a, n}=\sum_{n^{\prime} \in \mathcal{N}(n)} \bar{f}_{n, n^{\prime}}$ & $(1 d)$ \\
\hline \multicolumn{2}{|l|}{$\forall n \in \mathcal{N}, a \in \mathcal{A}(n)$} \\
\hline$\delta_{g} \geq\left(g_{a, n}-b_{a, n}\right) / g_{a, n}$ & $(1 \mathrm{e})$ \\
\hline$\delta_{G} \geq\left(G_{a, n}-B_{a, n}\right) / G_{a, n}$ & (1f) \\
\hline$g_{a, n} \leq b_{a, n} \leq 0 \leq B_{a, n} \leq G_{a, n}$ & $(1 \mathrm{~g})$ \\
\hline
\end{tabular}

would not be performed in one round, since access contracts are delivered within a certain delay after they were requested. The aggregation of contracts of the low voltage contributors should also be considered. A more realistic simulation would thus consider the legacy of access contracts.

In the day by day simulation of the system, the DSO uses the local baseline proposals to compute the dynamic access ranges $\left[d_{a, n, t}, D_{a, n, t}\right]$ of each agent $a$ for each period $t$ and bus $n$. To compute the dynamic ranges, the DSO first determines credible scenarios of deviation from the baseline proposal $p_{a, n, t}^{p}$, denoted $\underline{p}_{a, n, t}$ and $\bar{p}_{a, n, t}$. If $p_{a, n, t}^{p}$ is in the safe access range,

$$
\begin{aligned}
& \underline{p}_{a, n, t}=\max \left\{l_{a, n, t}, p_{a, n, t}^{p}-\beta_{a, n, t}\left|p_{a, n, t}^{b}\right|\right\} \\
& \bar{p}_{a, n, t}=\min \left\{L_{a, n, t}, p_{a, n, t}^{p}+\beta_{a, n, t}\left|p_{a, n, t}^{b}\right|\right\}
\end{aligned}
$$

where $\beta_{a, n, t}$ is a parameter quantifying the credible relative deviation. If $p_{a, n, t}^{p}$ is in the flexible access range, we set $\underline{p}_{a, n, t}=\bar{p}_{a, n, t}=p_{a, n, t}^{p}$. Two additional parameters, $\Delta d_{a, n, t}^{\max }$ and $\Delta D_{a, n, t}^{\max }$ give amount of curtailable power by the DSO and are computed by

$$
\begin{aligned}
\Delta d_{a, n, t}^{\max } & =\max \left\{0, l_{a, n}-p_{a, n, t}^{p}\right\} \\
\Delta D_{a, n, t}^{\max } & =\max \left\{0, p_{a, n, t}^{p}-L_{a, n}\right\}
\end{aligned}
$$

Together with the dynamic ranges, the DSO computes its upward and downward flexibility needs as a FSU, $r_{n, t}^{+}$and $r_{n, t}^{-}$ by solving optimization problem (4). These needs are computed as the maximum needs in each scenario. The positive parameters $\alpha_{n}^{+}$and $\alpha_{n}^{-}$characterize the flexibility potential at each bus and are obtained from the DSO knowledge of the system (this may for instance be part of the access contract procedure). The dynamic ranges are built using the solution

$$
\min \sum_{n \in \mathcal{N}}\left(\alpha_{n}^{+} r_{\mathrm{DSO}, n, t}^{+}+\alpha_{n}^{-} r_{\mathrm{DSO}, n, t}^{-}\right)
$$

subject to, $\forall n, n^{\prime} \in \mathcal{N}^{2}, t \in \mathcal{T}$,

$$
-C_{n, n^{\prime}} \leq \underline{f}_{n, n^{\prime}, t}, \bar{f}_{n, n^{\prime}, t} \leq C_{n, n^{\prime}}
$$

$\forall n \in \mathcal{N}, t \in \mathcal{T}$

$\sum_{a \in \mathcal{A}}\left(\underline{p}_{a, n, t} \Delta D_{a, n, t}+\Delta d_{a, n, t}\right)-\underline{r}_{n, t}^{-}+\underline{r}_{n, t}^{+}=\sum_{n^{\prime} \in \mathcal{N}(n)} \underline{f}_{n, n^{\prime}, t}$

$\sum_{a \in \mathcal{A}}\left(\bar{p}_{a, n, \bar{t}} \Delta D_{a, n, t}+\Delta d_{a, n, t}\right)-\bar{r}_{n, t}^{-}+\bar{r}_{n, t}^{+}=\sum_{n^{\prime} \in \mathcal{N}(n)} \bar{f}_{n, n^{\prime}, t}$

$r_{n, t}^{-} \geq \underline{r}_{n, t}^{-}, \bar{r}_{n, t}^{-}$

$r_{n, t}^{+} \geq \underline{r}_{n, t}^{+}, \bar{r}_{n, t}^{+}$

$\forall a \in \mathcal{A}, n \in \mathcal{N}, t \in \mathcal{T}$,

$$
\begin{aligned}
& \Delta d_{a, n, t} \leq \Delta d_{a, n, t}^{\max } \\
& \Delta D_{a, n, t} \leq \Delta D_{a, n, t}^{\max }
\end{aligned}
$$

of optimization problem (4) as

$$
\begin{aligned}
d_{a, n, t} & =\min \left\{l_{a, n}, p_{a, n, t}^{p}+\Delta d_{a, n, t}\right\} \\
D_{a, n, t} & =\max \left\{L_{a, n}, p_{a, n, t}^{p}-\Delta D_{a, n, t}\right\} .
\end{aligned}
$$

To obtain the final baselines communicated to the DSO and to the TSO, BRPs solve the same optimization problem they used to get their baseline proposal taking into account these dynamic ranges.

\section{RESULTS}

The interaction models are tested on a generic $11 \mathrm{kV}$ distribution network composed of 75 buses and hosting 22 distributed generation units [11]. Two interaction models are simulated under the same conditions for 365 days individually, which amounts to 32 hours of simulation by model. Each day is divided in 24 periods and simulated independently. 
Production data of the 22 generation units are taken from a production curve of 2013 scaled such that the maximum production reaches $64.2 \mathrm{MW}$ and the average is $16 \mathrm{MW}$. Distributed generation units are clustered into three portfolios, each managed by a different producer. The consumption of the 53 connected loads is built from the Belgian total load consumption of 2013 scaled to a mean of $10.8 \mathrm{MW}$ and a maximum of $21 \mathrm{MW}$. This consumption is divided in three parts, each belonging to one retailer. The consumption of a retailer is divided in a static part and a flexible part respectively accounting for $80 \%$ and $20 \%$ of the total consumption of the load. We assume that the TSO aims to contract a volume of flexibility equal to $2 \%$ of the total installed production capacity of the system in each period. The total activation request of the TSO is drawn using a zero mean Gaussian distribution with a variance equal to the target flexibility volume of the TSO. Energy prices are taken from the clearing of 2013 of the Belpex day-ahead energy market scaled to a mean of 53.64 $€ / M W h$ and a maximum of $93.03 € / M W h$, excluding the extreme $2.5 \%$ of the original data. The value of lost load taken into account in the case of shedding due to the tripping of a protection is set to $500 € / \mathrm{MWh}$ for the production and 1000 $€ / M W h$ for the consumption. The imbalance prices come from the Belgian TSO. A FSP not providing a contracted flexibility service or violating its dynamic range is penalized at $150 \%$ of the maximum imbalance price. The experiments are carried out on a computer equipped with an Intel Core i7-3770 CPU at $3.40 \mathrm{GHz}$ with $32 \mathrm{~GB}$ of RAM. The optimization problems are solved with SCIP.

First of all, we compare the proposed interaction model referred to as "dynamic baseline" with respect to the safest model of article [1], the Model 2, referred to as "restrictive" in this article. An example of results is given in Table I where the parameters $\beta_{a, n, t}$, defining the relative deviation from the baseline proposals, are arbitrarily fixed to $20 \%$. Table I provides daily results obtained from the simulation of the whole year. In this table, the welfare is defined as the sum of the surpluses and the costs of all actors minus a shedding cost proportional to the value of lost load. These results show a significant growth of the welfare of $47 \%$. This growth comes from an increase of the total production by $56 \%$ which directly impacts the revenues of the producers. The dynamic baselines interaction model requires shedding about $122 \mathrm{MWh}$ with a maximum of $11.48 \mathrm{MWh}$ on one day with the parameter $\beta_{a, n, t}=20 \%$. This amount of shedding is the result of actors deviating from their proposal in the safe access range. Note that they are free to deviate as they wish within this range, in this case even more than the $20 \%$ security margin of the DSO. To take into account these additional deviations, the DSO can easily use some margins and increase the relative deviation it considers. Fig. 4 shows that taking values greater than $30 \%$ avoids the shedding issues. Fig. 5 depicts the impact of the relative maximal deviation parameter on the welfare and the total production with respect to the restricted model. Even though the total production increases as $\beta_{a, n, t}$ decreases, the welfare reaches its maximum around $\beta_{a, n, t}=25 \%$ with a welfare increased by $47.57 \%$. Fig. 5 shows that the welfare is not as sensitive with respect to $\beta_{a, n, t}$ as we would expect. Therefore, a practical choice for this application would be $\beta_{a, n, t}=40 \%$ to make sure to avoid the shedding issues without notably impacting the welfare. With this value of the relative deviation, the welfare would be increased with respect to the restricted model by $47 \%$ and the total production by $55 \%$.

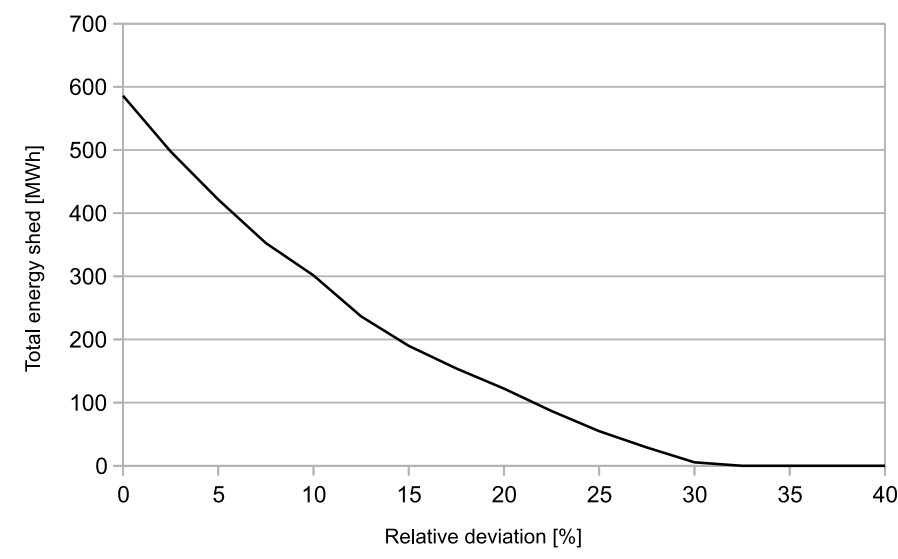

Fig. 4: Evolution of the yearly shed production with the relative maximal deviation parameter considered by the DSO.

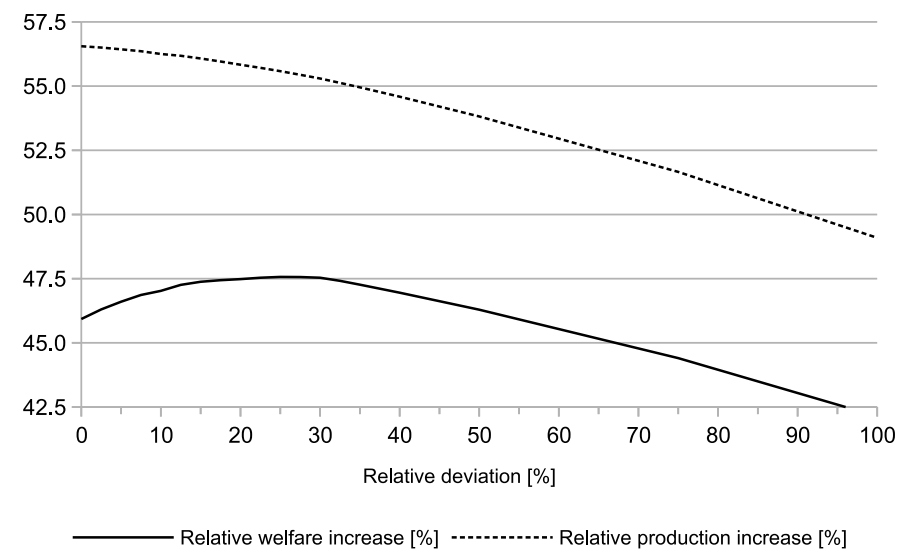

Fig. 5: Welfare and production increase as a function of the relative maximal deviation parameter considered by the DSO.

\section{CONCLUSION}

This article proposes an interaction model organizing the interactions between the actors of the distribution system exchanging flexibility. This new interaction model is based on dynamic access bounds to the network changing throughout the day and computed by the DSO. These bounds are computed using baseline proposals from the grid users at the medium voltage level and depends on an estimation of the DSO of the maximal deviation of the realization of the actor with respect to its proposal. This new interaction model is implemented in DSIMA and compared to the previous safest proposal, an interaction model restricting the grid users to a 
TABLE I: Comparison of the interaction models on one year.

\begin{tabular}{|c|c|c|c|c|c|c|c|}
\hline & \multicolumn{3}{|c|}{ Model "restricted" } & \multicolumn{3}{|c|}{ Model "dynamic baselines" } & \multirow[t]{2}{*}{ Units } \\
\hline & $\min$ & mean & $\max$ & $\min$ & mean & $\max$ & \\
\hline Welfare & -7727.27 & 27410.94 & 68434.42 & -7726.54 & 40426.38 & 151596.04 & $€$ \\
\hline Shedding costs & 0 & 0 & 0 & 0 & 167.31 & 5740.86 & $€$ \\
\hline DSOs costs & 0 & 0 & 0 & 0 & 0 & 0 & $€$ \\
\hline TSOs surplus & 831.52 & 2878.34 & 3849.78 & 831.52 & 2877.74 & 3849.78 & $€$ \\
\hline Producers surplus & 0 & 24005.32 & 68265.06 & 0 & 37188.73 & 151426.68 & $€$ \\
\hline Retailers surplus & -14750.43 & 527.28 & 9852.33 & -14750.43 & 527.22 & 9852.33 & $€$ \\
\hline Total production & 0 & 247.33 & 622.26 & 0 & 385.42 & 1333.51 & MWh \\
\hline Total consumption & -489.93 & -257.07 & -39.95 & -489.93 & -257.07 & -39.95 & MWh \\
\hline Total imbalance & 0.15 & 1.4 & 5.11 & 0.15 & 1.73 & 15.16 & MWh \\
\hline Max. imbalance & -0.6 & -0.13 & 0.3 & -7.09 & -0.36 & 0.3 & MW \\
\hline Total usage of flex. & 0 & 14.94 & 65.1 & 0 & 14.92 & 65.1 & MWh \\
\hline Total energy shed & 0 & 0 & 0 & 0 & 0.33 & 11.48 & MWh \\
\hline
\end{tabular}

very restrictive but safe access range. Results show that this new model achieves better performances than the restrictive model, allowing to safely increase by $55 \%$ the amount of distributed generation in the network and the welfare by $42.5 \%$.

The work presented in this paper could be extended along several lines, for instance by computing fair access ranges to the network taking into account the current system situation and future evolution; refining the modeling level by considering more detailed agents, considering the analysis in a stochastic environment and alternating current power flow equations; studying dynamics of the system, such as the entry or exit of new players or production units; and finally comparing the proposed interaction models to network reinforcement decisions without change of interaction model.

\section{ACKNOWLEDGMENTS}

This research is supported by the public service of Wallonia - Department of Energy and Sustainable Building within the framework of the GREDOR project.

\section{REFERENCES}

[1] S. Mathieu, Q. Louveaux, D. Ernst, and B. Cornélusse, "Dsima: A testbed for the quantitative analysis of interaction models within distribution networks," Sustainable Energy, Grids and Networks, vol. 5, pp. 78 -93, 2016, ISSN: 2352-4677.

[2] L. Kane and G. Ault, "A review and analysis of renewable energy curtailment schemes and principles of access: Transitioning towards business as usual," Energy Policy, vol. 72, pp. 67-77, 2014.

[3] R. Currie, G. W. Ault, C. Foote, and J. R. McDonald, "Active power-flow management utilising operating margins for the increased connection of distributed generation," IET generation, transmission \& distribution, vol. 1, no. 1, pp. 197-202, 2007.
[4] K. Heussen, D. E. M. Bondy, J. Hu, O. Gehrke, and L. H. Hansen, "A clearinghouse concept for distributionlevel flexibility services," in 4th Innovative Smart Grid Technologies Europe, IEEE/PES, 2013.

[5] P. Bach Andersen, J. Hu, and K. Heussen, "Coordination strategies for distribution grid congestion management in a multi-actor, multi-objective setting," in $3 r d$ Innovative Smart Grid Technologies Europe, IEEE/PES, 2012.

[6] L. Merciadri, S. Mathieu, D. Ernst, and Q. Louveaux, "Optimal assignment of off-peak hours to lower curtailments in the distribution network," in 5th Innovative Smart Grid Technologies Europe, IEEE/PES, 2014.

[7] S. Mathieu, D. Ernst, and Q. Louveaux, "An efficient algorithm for the provision of a day-ahead modulation service by a load aggregator," in 4th Innovative Smart Grid Technologies Europe, IEEE/PES, 2013.

[8] E. Georges, B. Cornélusse, D. Ernst, Q. Louveaux, V. Lemort, and S. Mathieu, "Direct control service from residential heat pumps aggregation with specified payback," in Proceedings of the 19th Power Systems Computation Conference (PSCC), IEEE, 2016.

[9] S. S. Oren, "A historical perspective and business model for load response aggregation based on priority service," in System Sciences (HICSS), 2013 46th Hawaii International Conference on, IEEE, 2013, pp. 2206-2214.

[10] K. Margellos and S. Oren, "A fuse control paradigm for demand side management: Formulation and stochastic pricing analysis," in American Control Conference (ACC), 2015, IEEE, 2015, pp. 5672-5677.

[11] SEDG, Uk generic distribution system (ukgds) project, 2010. [Online]. Available: www.sedg.ac.uk. 